\title{
PLANEAMIENTO ESTRATÉGICO CON BSC EN UN MODELO DE EXCELENCIA EN LA EMPRESA TEXTIL HAKENI Y MEJORA CONTINUA
}

\begin{abstract}
María Celina Huamán Mejía*
RESUMEN

El estudio presenta un informe de diseño de planeamiento estratégico con BSC, en un modelo de excelencia en la empresa textil Hakeni y mejora continua, la excelencia empresarial, interacción con el medio y la internacionalización desplegada en un conjunto de indicadores de aprendizaje, gestión de procesos, clientes, mercado y finanzas se relaciona directamente con la mejora continua de la empresa, muestra una información muy exacta en el contexto de un modelo de excelencia ya que a partir del perfil organizacional se determinan los desafíos estratégicos en el plan.

El Plan Estratégico con BSC tiene elementos para la ejecución y seguimiento como son: un mapeo estratégico en cuatro perspectivas, cuatro proyectos o iniciativas: a) Proyecto de implementación de gestión por procesos, b) Proyecto de implementación y gestión por competencias, c) Proyecto de plan de marketing y d) Proyecto de implementación de sistema de información; cada uno de ellos con impactos en el desarrollo de la empresa. El estudio demuestra una mejora continua en la gestión con las nuevas herramientas diseñadas a la medida, herramienta delineada de acuerdo a las necesidades y características de la empresa Hakeni.
\end{abstract}

praxiscuality@gmail.com

Palabras Claves: plan estratégico, balanced scorecard, mejora continua.

\begin{abstract}
The study presents a Design Report with BSC Strategic Planning in a model of Excellence in Textile Enterprise Hakeni and continuous improvement, business excellence, interaction with the environment and internationalization deployed on a set of indicators of learning, process management, customers, market and finance is directly related to the continuous improvement of the company, shows a very accurate information in the context of a model of excellence and that from the organizational profile identifies the strategic challenges in the Plan.

The Strategic Plan has strategic elements for BSC implementation and monitoring such as: A strategic mapping four perspectives, four projects or initiatives: a) Project management of implementation processes, b) implementation and project management skills, project Marketing plan and Project implementation information System each with an impact on the development of the company. The study shows that continuous improvement is demonstrated in the management with new tools designed to measure, outlined tool according to the needs and characteristics of the company Hakeni.

Keywords: Strategic Plan, Balanced Scorecard, Continuous Improvement.
\end{abstract}

\footnotetext{
Magíster en administración, Licenciada en Administración, Docente Asociada de la Facultad de Ciencias Administrativas de la UNMSM.
} 


\section{INTRODUCCIÓN}

Un empresario puede ser concebido como un emprendedor que visualiza oportunidades de negocio y las aprovecha sobre la base de esfuerzo y de su capacidad de interacción exitosa con el entorno. Se puede afirmar que existen dos tipos de empresarios: los que opinan abiertamente por esta forma de vida y los que, por las circunstancias que se les presentan, ingresan al mundo empresarial o " devienen" empresarios. El planeamiento estratégico resulta fundamental para empresas de ambos grupos que se perfilan a un modelo de gestión por resultados. Este ha sido diseñado con base en principios internacionalmente aceptados como inspiradores de una gestión de excelencia. Buenos resultados son la meta de todo proceso, los resultados permiten evaluar el cumplimiento de los objetivos planteados durante la planificación, además todo resultado revela el nivel de eficacia, efectividad y eficiencia, en el desempeño de los procesos. ¿Cómo debo hacer el despliegue de la dirección estratégica de la empresa?

Los doctores David Norton y Robert Kaplan nos dan una respuesta metodológica a este tema, describiendo con sumo detalle de qué manera una organización adopta ese enfoque integrado, que fue utilizado por numerosas organizaciones con resultados excepcionales. Sin embargo, hacía falta los "Mapas Estratégicos", para proporcionarnos total claridad acerca de uno de los aspectos más difíciles de la metodología del BSC: describir la estrategia de una organización utilizando diferentes perspectivas y desagregando de la misma en objetivos individuales relacionados entre sí por una lógica de causa y efecto. La "Propuesta de Valor", por ejemplo, componente fundamental a ser analizado en el mapa estratégico, representa gran parte del valor agregado del servicio de formación profesional diferenciado que impartimos en los programas de pre y postgrado, es también una gran oportunidad frente a un mercado tan competitivo.

Pensamos que este trabajo es de vital importancia para todas las empresas textiles que quieran gestionarse estratégicamente, tratando de minimizar el riesgo de una mala ejecución de sus objetivos fundamentales.

\section{MÉTODOS}

La presente investigación se basó principalmente en la revisión de la literatura general acerca del modelo de excelencia en la gestión y el cuadro de mando integral, posteriormente la literatura especializada acerca de lo dirección estratégica, un nuevo marco para describir y alinear las cuatro perspectivas: aprendizaje y crecimiento -capital humano, capital de información y capital organizacional- en los procesos estratégicos y los objetivos de la perspectiva interna. Procesos internos y financieros. La metodología que se sigue es estrictamente la metodología Norton y Kaplan, y modelo de excelencia en la gestión en el marco del criterio Planeamiento Estratégico. El enfoque de la investigación es cualitativa, de tipo descriptivo, observacional-relacional.

Universo. Ámbito: empresa textil Hakeni.

La Muestra. A) Para el capital humano: La muestra es de tipo no probabilística por conveniencia para los actores clave.

Unidad de Análisis: Se define el universo por la unidad de análisis a todos los colaboradores que trabajan en el nivel táctico y de planta

\section{VARIABLES}

\section{Mejora Continua}

»Capital humano

»Capital organizacional y

» Capital de información

\section{Plan Estratégico con Balanced Scorecard}

» Perspectiva: innovación y desarrollo

» Perspectiva: procesos internos

» Perspectiva: Clientes

Perspectiva: Financiera

\section{INSTRUMENTOS}

a) Autoevaluación en base al modelo de Excelencia en la Gestión 2008.

b) Análisis estratégico del sector textil

Análisis de los datos: se tomó

a) como fuente primaria

b) como fuente secundaria. 


\section{RESULTADOS}

La investigación describe de qué forma la empresa desarrolla el indicador es del modelo en las perspectivas clave del BSC. Asimismo muestra que los factores explicativos de la mejora continua se explican en 19 subcriterios y son: (Ver Anexo N. ${ }^{1}$ )

\section{Código de Postulación Iniciales del Evaluador}

Para iniciar un proceso de evaluación, revise el Perfil Organizacional del postulante. Liste los factores clave de la organización usando los encabezados en el orden que se presentan en la sección "Preparación del Perfil Organizacional".

\section{Ambiente Organizacional}

» Una empresa de diseño y comercialización de prendas de vestir para el segmento juvenil distribución y comercialización en una sola ubicación (San Juan de Miraflores, Lima Perú), se divide entre productos: Jeans, Poleras y Polos.

- Los empleados son denominados "trabajadores".

- 4 de nivel directivo

- 13 trabajadores en un ambiente no sindicalizado, nombrados a tiempo completo y 11 operarios. Total : 29

- Trabajadores de contabilidad, ventas, cobranza, almacén (33.33\%).
- Todos los trabajadores tienen educación secundaria, superior, técnico y estudios universitarios

- Experiencia: el trabajo requiere competencias y experiencia técnica significativa.

» Tecnologías más importantes:

- Software de contabilidad-SIIGO (Estándar)

- Software diccionario de datos (implementación de reportes - SIIGO)

- Software control horario de trabajo

- Software planilla de movilidad (gestión de gastos por movilidad)

- Software, movimientos de Bancos

- Sistema Intranet

\section{Relaciones Organizacionales}

» Ventas principalmente al mercado local (Lima Metropolitana). Los clientes son principalmente clientes recurrentes. Grandes clientes, medianos y pequeños. Proveedores clave de productos principales.

\section{Ambiente Competitivo}

» Alto grado de rivalidad en la industria de la construcción. Industria con perspectivas de crecimiento $30 \%$ anual (2008)

\section{Desafíos Estratégicos}

» Generar valor

» Optimización financiera tributaria

» Asegurar niveles de servicio

» Reconocimiento del mercado objetivo

» Diseño, implementación y mejora continua de procesos que agregan valor y de soporte

» Capital humano competente

» Incorporación de tecnologías

\section{Sistema de Mejora del Desempeño}

Desarrollar filosofía de calidad y mejora continua, adoptar mejores prácticas de gestión, formar cuadros ejecutivos. 


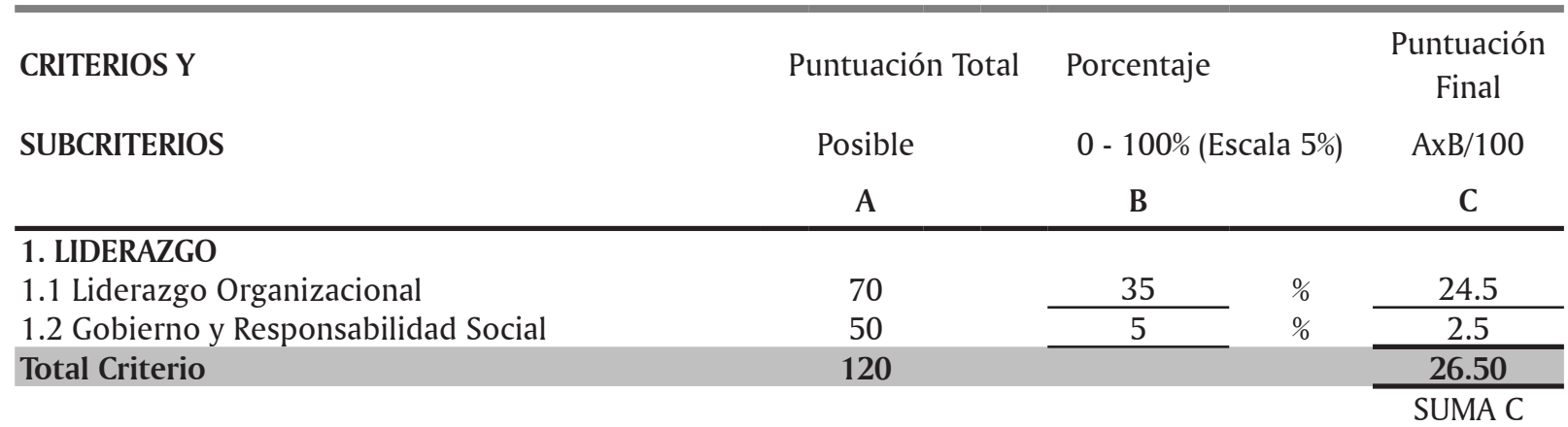

\section{PLANEAMIENTO ESTRATÉGICO}

2.1 Desarrollo de Estrategias

2.2 Despliegue de Estrategias

Total Criterio
20

\begin{tabular}{llll}
40 & & $\%$ & 12 \\
\cline { 2 - 3 } 45 & 15 & $\%$ & 6.8 \\
\hline 85 & & $\frac{18.8}{20}$
\end{tabular}

\section{ORIENTACIÓN HACIA EL CLIENTE Y EL MERCADO}

3.1 Conocimiento del Cliente y del Mercado

3.2 Relaciones con el Cliente y Satisfacción

Total Criterio

\begin{tabular}{llll}
40 & 30 & $\%$ & $\frac{12}{25}$ \\
\cline { 2 - 3 } & & $\%$ & $\frac{11.3}{25.3}$ \\
\cline { 2 - 3 } & & & $\frac{23}{\text { SUMA C }}$
\end{tabular}

\section{MEDICIÓN, ANÁLISISY GESTIÓN DEL CONOCIMIENTO}

4.1 Medición, análisis y revisión del desempeño or-

ganizacional

4.2 Información y gestión del conocimiento

Total Criterio

\begin{tabular}{llll}
45 & 35 & $\%$ & $\frac{15.8}{6.8}$ \\
\hline 90 & $\%$ & $\frac{22.6}{\text { SUMA C }}$
\end{tabular}

\section{ORIENTACIÓN HACIA EL PERSONAL}

5.1 Sistemas de Trabajo 35

5.2 Aprendizaje y Motivación de los Trabajadores 25

5.4 Bienestar y Satisfacción de los Trabajadores 25

Total Criterio

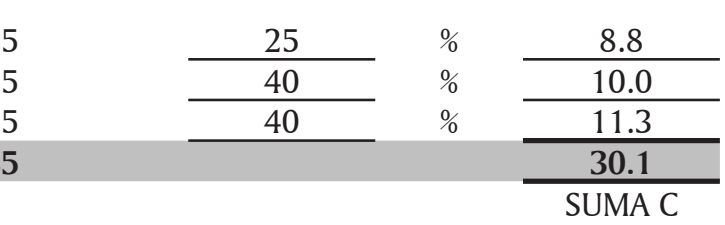

\section{GESTIÓN DE PROCESOS}

6.1 Procesos de Creación de Valor

6.2 Procesos de Soporte y Planeamiento Operacional

Total Criterio

\begin{tabular}{|c|c|c|c|}
\hline 45 & 15 & $\%$ & 6.75 \\
\hline 40 & 15 & $\%$ & 6.75 \\
\hline 85 & & & 13.5 \\
\hline
\end{tabular}

\section{RESULTADOS}

7.1 Resultados de Orientación hacia el Cliente 70

$\begin{array}{ll}7.2 \text { Resultados de Productos y Servicios } & 100\end{array}$

7.2 Resultados Financieros y de Mercado 70

7.3 Resultados del Personal 70

7.4 Resultados de Eficiencia Organizacional 70

7.5 Resultados de Liderazgo y Responsabilidad Social

$\begin{array}{lc}\text { Total Criterio } & 450\end{array}$

TOTAL (D) $\quad \underline{1000}$

Elaboración: propia

\begin{tabular}{|c|c|c|}
\hline 25 & $\%$ & 17.5 \\
\hline 35 & & 35.0 \\
\hline 20 & $\%$ & 14.0 \\
\hline 35 & $\%$ & 24.5 \\
\hline 30 & $\%$ & 21 \\
\hline \multirow[t]{4}{*}{15} & $\%$ & 10.5 \\
\hline & & 123 \\
\hline & & SUMA C \\
\hline & & 258.3 \\
\hline
\end{tabular}

El estudio de análisis estratégico demuestra que el sector es muy competitivo y el mercado externo es el ideal, formar competencias que alinee a las exigencias del mercado internacional es una constante en esta empresa, además se muestra una tendencia favorable (Ver Anexo 2). 


\section{ANÁLISIS ESTRATÉGICO DE LA INDUSTRIA TEXTIL EN EL PERÚ ANEXO N.o 02}

Indicadores Macroeconómicos

- $\quad \mathrm{Al} 07 / 01 / 2008$ las reservas internacionales netas de Perú llegaron a 28305000000 dólares americanos.

- El factor Riesgo País se ubicó en 189 pbs al 09/01/2008.

- La Tasa de Interés de Referencia subió de $5,00 \%$ a $5,25 \%$.

\begin{tabular}{|c|c|c|c|}
\hline INDICADOR & FECHA & UNIDAD & VALOR \\
\hline Reservas Internacionales netas & $7 / 01 / 2008$ & millones US\$ & $28,305,00$ \\
\hline Riesgo País & $9 / 01 / 2008$ & pbs & 189,00 \\
\hline Tipo de cambio & $9 / 01 / 2008$ & soles por US\$ & 2,95 \\
\hline Tasa de interés corporativo & $9 / 01 / 2008$ & $\%$ & 4,99 \\
\hline Inflación & diciembre & $\%$ & 0,45 \\
\hline Superávit comercial & noviembre & millones US\$ & 580,00 \\
\hline Superávit económico & noviembre & millones & 688,00 \\
\hline PBI & octubre & $\%$ & $10.4+$ \\
\hline $\begin{array}{c}\text { Superávit cuenta corriente } \\
\text { Superávit }\end{array}$ & 3 trimestre & millones US\$ & 270,00 \\
\hline $\begin{array}{c}\text { Variación tasa de interés } \\
\text { referencial }\end{array}$ & $10 / 01 / 2008$ & $\%$ & 5,0 a 5,25 \\
\hline Fuente: BCRP & & \multicolumn{2}{|c|}{$11 / 01 / 2008$} \\
\hline
\end{tabular}

PRODUCTO BRUTO INTERNO (PBI)

PBI Y DEMANDA INTERNA
(Variaciones porcentuales anuales)

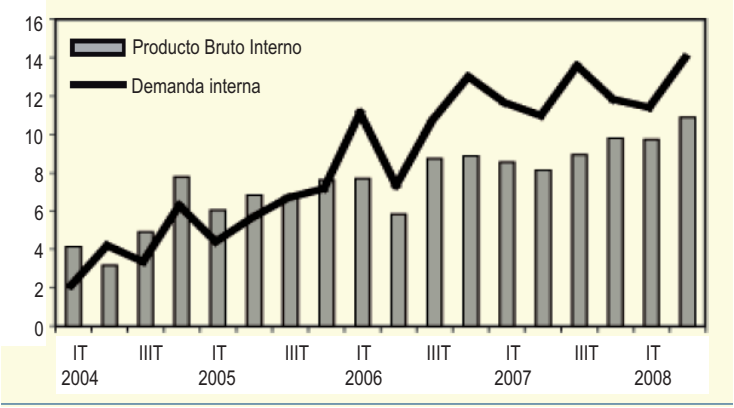

ECONOMÍAS MUNDIALES RELACIONADAS CON PERÚ

Desaceleración pronunciada de Economias Desarrolladas.

China con crecimiento menor al 2008

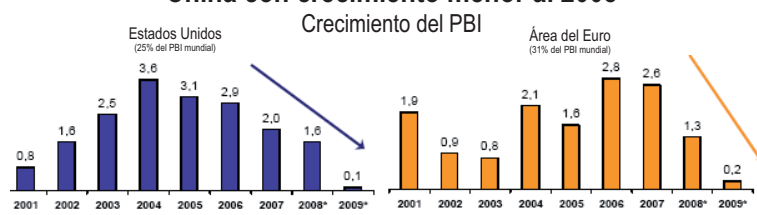

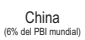

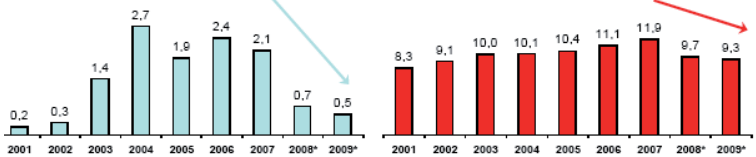

\section{EXPORTACIONES}

Participación en las exportaciones del Perú

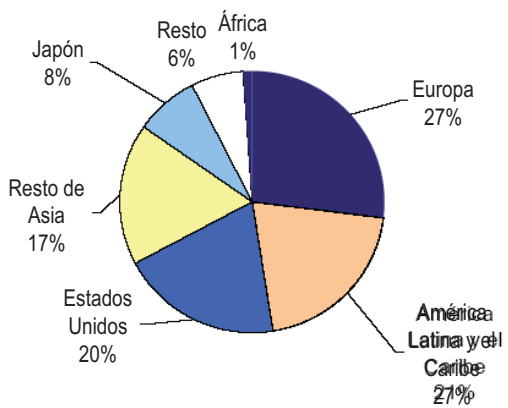

\section{EXPORTACIÓN DEL SECTOR CONFECCIÓN Y TEXTILES}

\section{DINÁMICA EXTERNA}

\section{Entorno Internacional}

» China constituyó el 30,8\% de las importaciones de confecciones en EEUU en 2007.

»Importaciones de confecciones en EEUU (en millones de US\$ por países, promedio 20052007).

» Los productos chinos siguen ganando terreno en la categoría 338/339 en EEUU

»Importaciones de EEUU en las categorías 338/339 ( en millones US\$: Anual 2005-2007, Ene-Mar 2007-2008, var\%).

» Las ventas minoristas de prendas de vestir en EEUU crecieron 4\% entre el 2002-2007.

» EEUU: Estacionalidad de las importaciones de confecciones (en millones de US\$: 2004-2007, mensual).

\section{Dinámica Interna}

Dinámica Local

» Producción del sector de confecciones creció 7,2\% en el 2007.

» Expansión del área sembrada incrementó la producción de algodón en el 2007.

» Algodón: producción local vs importación (en miles de TM: 2002-2007, proyectado 2008, anual).

» Exportaciones crecieron 15,6\% en el 2007.

» Exportación de prendas de vestir y otras confecciones (en millones de US\$: 2002- 2007, proyectado 2008, anual; Var \%).

» Exportación de prendas de vestir y otras con- 
fecciones (en millones de US\$: 2006- 2008, mensual).

» Exportaciones de prendas de vestir de tejido plano crecieron $64,4 \%$ en el primer trimestre del 2008.

» Exportación de vestuario y otras (en millones de US\$: 2006-2007, anual; Ene- Mar 20072008).

Evolución de la inflación: Enero 2002-Febrero 2008 (variación porcentual 12 meses)

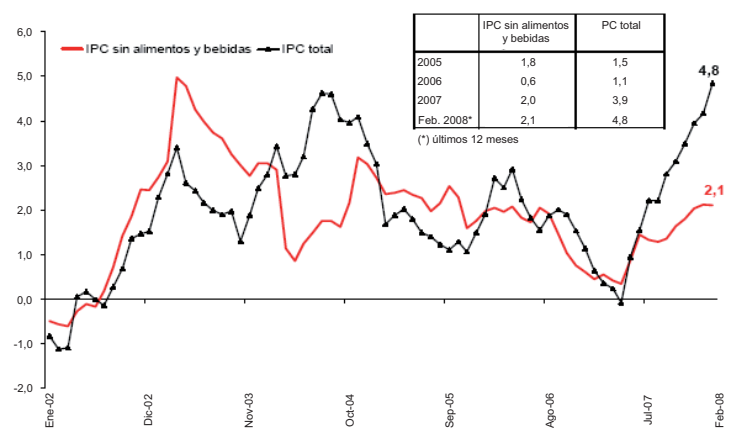

$1,0 \quad 3,0$

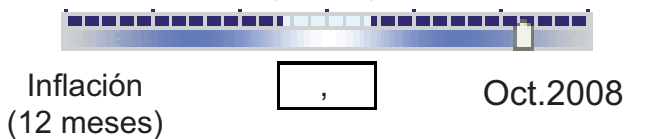

» Exportaciones a Europa representaron el 9,2\% del total de los envíos durante el primer trimestre del 2008.

》Destino de las exportaciones de confecciones (US\$: 2005-2007).

» Topy Top siguió liderando las exportaciones de prendas de tejido en el 2007.

» Principales exportadores de confecciones (en millones de US\$: 2006-2007, anual, Ene-Mar 2007-2008).

\section{Dinámica Empresarial}

» Perú empieza a posicionarse con confecciones de alto valor

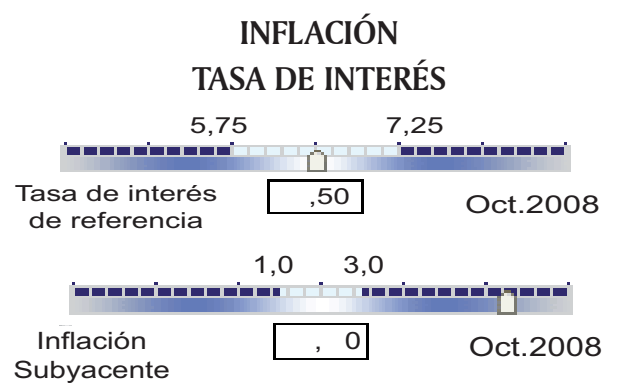

ANÁLISIS EXTERNO

\section{OPORTUNIDADES}

- Uno de los proyectos que está realizando el Grupo Romero con algunas comunidades andinas es el de la creación de un nuevo tipo de algodón, el cual, según estudios, tiene mejores propiedades que los que tiene el algodón Pima o Tanguis.

- Las alianzas estratégicas que puedan realizar con talleres textiles (tercerización).

- Posible tratado de libre comercio con Japón, permitirá obtener las máquinas textiles a menor precio (en el caso implementen su taller).

- El mercado que conforman los jóvenes universitarios $E M O$, prefieren utilizar vestimenta de color negro.

- El proyecto le interese a una empresa grande con quien haría una alianza estratégica.

\section{AMENAZAS}

- Los tratados de libre comercio con los diferentes países, que comercializan sus productos (polos) dentro de nuestro país a menor precio.

- La crisis económica que se está suscitando en los EEUU afectará a la economía peruana debido a la globalización.

- Las grandes empresas como Saga Falabella, Ripley, Topi Top e incluso tiendas de Gamarra, tienen un mercado fijo, el cual también incluye el mercado objetivo de Hakeni.

- Competencia ilegal, productos piratas (copia de los nuevos diseños).

- La cultura de los consumidores (personas con alto poder adquisitivo).

- Preferencia a la utilización de marcas exclusivas.

- Comprar sus productos en centros de mayor status, como Jockey Plaza.

- Buscan diferenciarse y la empresa no ofrece variedad de diseños.

- Los gustos de los consumidores (Mercado Femenino).

- Solo ofrecen al mercado dos colores: blanco y negro.

- El corte del polo es muy simple, no ofrece elegancia ni variedad.

- Los consumidores adquieren su vestimenta 
guiándose de la estacionalidad. Por ejemplo, en verano son pocas las personas que utilizan ropa negra.

- La imposibilidad de utilizar logos de instituciones educativas privadas y publicas, sin previa autorización de las mismas, por ejemplo, Universidad del Pacífico, Pontificia Universidad Católica y otras.

El Plan estratégico con BSC se muestra en el (Anexo 3).

\section{CONCLUSIONES}

El estudio permite señalar que el plan estratégico con BSC es un instrumento de gestión que exige el contexto actual y tienen una orientación clara a la mejora continua.

El estudio muestra que el plan estratégico con BSC propuesto, crea una nueva mentalidad para gestionar el desarrollo y el despliegue de los indicadores financieros, de procesos, clientes y financieros. Gestionar con ese enfoque conduce a la mejora continua en las perspectivas mencionadas.

Vimos que los activos se han vuelto decisivos para la creación sustentable de valor. En la perspectiva de aprendizaje y crecimiento del BSC de la empresa, la función de alinear los activos intangibes con su estrategia. Esta perspectiva contiene los objetivos e indicadores de tres componentes de activos intangibles esenciales para implementar cualquier estrategia.

\section{BIBLIOGRAFÍA}

http://www.gruporomero.com.pe/Memoria\%20 Grupo\%20Romero\%202005\%20part1.pdf

http://www.bcrp.gob.pe/bcr/dmdocuments/PolMon/Archivos/RI_2008_03.pdf

http://www.gruporomero.com.pe/memoriaGR22006.pdf

http://economiayfinanzasdeperu.blogspot.com/ search/label/peru

http://www.bcrp.gob.pe/bcr/dmdocuments/Publicaciones/Discursos/PD_2008017.pdf

http://www.bcrp.gob.pe/bcr/dmdocuments/Estadistica/IndicaTrim/Triptico.pdf

http://www.bcrp.gob.pe/bcr/dmdocuments/Publicaciones/Discursos/PD 2008006.pdf

http://www.maximixe.com/informes/confecciones/ confecciones.pdf

http://www.educ.ar/educar/Sobre\%20gustos $\% 20 \%$ BFno\% 20 se $\% 20$ discute $\% 20$. html?uri =urn:kbee:02a 8 b430-5a $5 \mathrm{a}$ $11 \mathrm{dd}-\mathrm{ba} 24-00163$ e $000038 \&$ page uri = urn:kbee:ff9221c0-13a9-11dc-b8c40013d43e5fae

http://www.elcomercio.com.pe/ediciononline/ HTML/2008-02-21/Peru-busca-incluiracuerdo-libre-comercio-agenda-Japon.html 